\title{
Plasma enhance drug sensitivity to bortezomib by inhibition of cyp1a1 in myeloma cells
}

\author{
Dehui Xu ${ }^{1}$, Qingjie Cui ${ }^{2}$, Yujing Xu ${ }^{1}$, Zeyu Chen ${ }^{1}$, Wenjie Xia ${ }^{1}$, Yanjie Yang ${ }^{3}$, Dingxin Liu ${ }^{1}$ \\ ${ }^{1}$ State Key Laboratory of Electrical Insulation and Power Equipment, Centre for Plasma Biomedicine, ${ }^{2}$ The School of Life Science and Technology, \\ ${ }^{3}$ Department of Cardiovascular Medicine, First Affiliated Hospital of the Medical School, Xi'an Jiaotong University, Xi'an 710049, China \\ Contributions: (I) Conception and design: D Xu, Y Yang; (II) Administrative support: D Xu, D Liu; (III) Provision of study materials or patients: \\ Y Yang, D Xu; (IV) Collection and assembly of data: Q Cui, Z Chen; (V) Data analysis and interpretation: Y Xu, W Xia; (VI) Manuscript writing: All \\ authors; (VII) Final approval of manuscript: All authors. \\ Correspondence to: Dehui Xu. Centre for Plasma Biomedicine, Xi'an Jiaotong University, 99 Yanxiang Road, Xi'an 710054, China. \\ Email: dehuixu@hotmail.com; Yanjie Yang. Department of Cardiovascular Medicine, First Affiliated Hospital of the Medical School, Xi'an Jiaotong \\ University, 277 Yanta West Road, Xi’an 710061, China. Email: 576059172@qq.com.
}

\begin{abstract}
Background: Drug resistance is one of the major problems encountered in clinical therapy of multiple myeloma treatment. Combination treatment with several drugs may increase the sensitivity and overcome drug resistance.

Methods: Here, we combined chemotherapy with a newly developed technology, cold atmospheric plasma, to enhance drug sensitivity.

Results: We found that plasma treatment had a synergistic anti-cancer effect with a first line drug (bortezomib). Based on our previous study, we further found that plasma treatment could inhibit Notch pathway and down-regulate cyp1a1 expression and enzyme activity, which contributing to the enhanced drug sensitivity to bortezomib after combination of bortezomib with gas plasma.
\end{abstract}

Conclusions: Our results showed a new strategy to overcome drug resistance by combination of traditional chemotherapy with cold atmospheric plasma.

Keywords: Cold atmospheric plasma; cyp1a1; drug resistance; multiple myeloma (MM); notch

Submitted Jun 18, 2019. Accepted for publication Oct 11, 2019.

doi: $10.21037 /$ tcr.2019.10.43

View this article at: http://dx.doi.org/10.21037/tcr.2019.10.43

\section{Introduction}

Multiple myeloma (MM), an incurable B cell disease, is characterized by the accumulation of malignant plasma cells in patients' bone marrow (BM) (1). Patients with MM are at an increased risk of developing infections, anemia, thrombocytopenia, renal failure, and bone disease $(2,3)$. Drug resistance is one of the major problems in MM clinical therapy (4). The interactions between MM cells and the BM microenvironment is considered to play a critical role in MM drug resistance (5). In between, the Notch pathway is important for MM cell growth, migration and drug resistance $(6,7)$. We have demonstrated that Notch activation could induce drug resistance to bortezomib in murine and human MM cells by up-regulating cyp1a1, which was involved in drug metabolism (8). In this study, we used a new technology, called cold atmosphere plasma (CAP), to treat MM cells $(9,10)$. CAP is mainly consisted of free electron and charged ions, which generates reactive oxygen species (ROS) and reactive nitrogen species (RNS) that could have various biological effects $(11,12)$. Thus, gas plasma has been applied in many biomedical fields such as sterilization, dentistry, cosmetology, wound healing, skin disease and cancer therapy (13-15). Combination of different drugs or therapy for a better anti-cancer effect is a common strategy in cancer therapy. Here, we demonstrated for the first time that gas plasma and bortezomib had a synergistic reduction of cell viability in myeloma cells. 

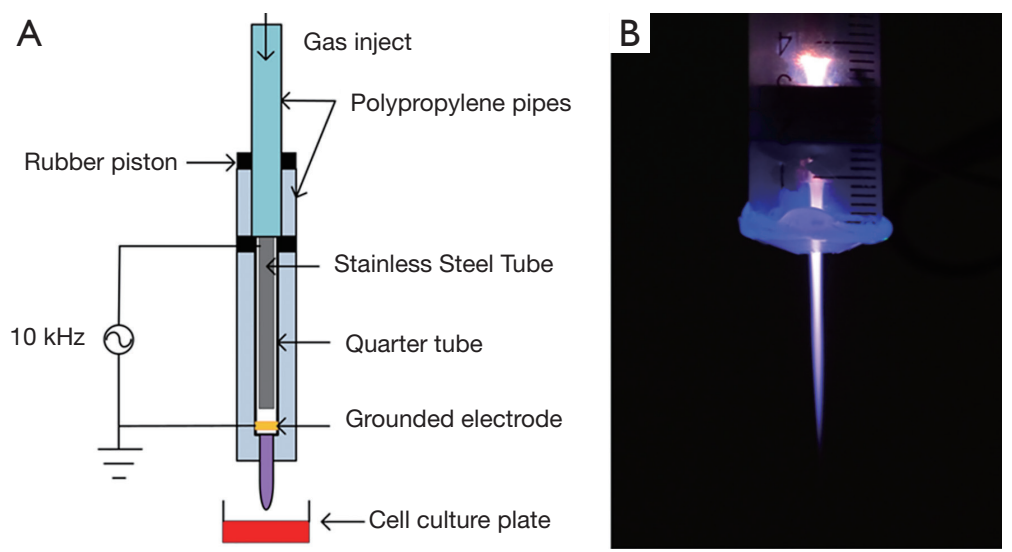

Figure 1 Schematic diagram of gas plasma generation. (A) The structure diagram of a gas plasma jet. (B) The discharging image of He plasma jet.

Gas plasma could enhance the sensitivity to bortezomib in myeloma cells, by inhibiting Notch signaling and cyp1a1, which could be applied as a new combination treatment for a better anti-cancer effect and a lower drug side effect.

\section{Methods}

\section{Atmospheric pressure plasma jet (APPZ) generation system.}

In this study, CAP was produced by a plasma jet device, the schematic diagram of the device structure was shown in Figure 1A. It was modified based on a needle. An inner diameter of $4 \mathrm{~mm}$ stainless steel tube was used as a highvoltage electrode and gas inlet. The ground electrode was a $10 \mathrm{~mm}$ long copper sheet, which wrapped around the quartz tube at a distance of $10 \mathrm{~mm}$ from the nozzle. The plasma generation system is consist of a gas flow controller, a high-voltage power supply, oscilloscope, and the above mentioned APPJ device. It was powered by a $10 \mathrm{kHz}$ sinusoidal power supply at $8 \mathrm{kV}$ peak-to-peak voltage and the $\mathrm{He}$ gas flow was maintained at 2 SLM. The plasma plume could directly contact with the cell medium surface.

\section{Optical emission spectroscopy}

Optical emission spectra (OES) was detected by an Andor SR-750i grating monochromator (grating grooving 1,200 lines $\mathrm{mm}^{-1}$ ) within a wavelength range of $300-800 \mathrm{~nm}$. The optical fiber was oriented up the APPJ radial direction at a distance of 1 and $2 \mathrm{~cm}$ from the APPJ nozzle.

\section{Cell culture and plasma treatment}

LP-1 MM cell line was used in this study and the details could be found in our previous work (16). Cells were cultured in Roswell Park Memorial Institute (RPMI) 1640 medium supplemented with $10 \%$ foetal calf serum, $100 \mathrm{U} / \mathrm{mL}$ penicillin, and $50 \mu \mathrm{g} / \mathrm{mL}$ streptomycin (Corning, Ithaca, NY, USA) in an incubator (Thermo Scientific, Waltham, MA, USA) containing a humidified atmosphere of $5 \% \mathrm{CO}_{2}$ at $37{ }^{\circ} \mathrm{C}$. Cells were refreshed $24 \mathrm{~h}$ before performing experiments. For plasma treatment, $2 \times 10^{5}$ cells were cultured in 24 well plates in $300 \mu \mathrm{L}$ RPMI1640 complete medium and were treated with plasma jet $1.5 \mathrm{~cm}$ away from the bottom of the plates. After treatment, cells were continually cultured for further experiments. Ethics approval was not required because no clinical experiments were involved in our paper.

\section{Cell viability assay}

In this study, we used CellTiter-Glo Assay kit (Promega, Madison, WI, USA) to detect the cell viability, which based on the quantitative determination of ATP in living cells. $100 \mu \mathrm{L}$ of cells and $100 \mu \mathrm{L}$ of Cell-Titer-Glo reagent were mixed into the opaque-walled multi-well plate, then cells were incubated at room temperature for $10 \mathrm{~min}$. The luminescence was determined using the microplate reader (Thermo Scientific, USA) with the protocol of "Luminometric" measurement. 


\section{Extracellular and intracellular ROS detection}

The ROS levels were monitored using CMH2-DCFDA (Invitrogen, USA) following the manufacturer's instruction. After plasma treatment for $24 \mathrm{~h}, 10 \mu \mathrm{M}$ of ROS dye was incubated with cells for $30 \mathrm{~min}$ at $37^{\circ} \mathrm{C}$. Extracellular ROS was measured with a microplate reader (Thermo Scientific) with excitation/emission at $485 / 530 \mathrm{~nm}$ using the protocol for "Fluorometric" measurement. Then, the cells were washed three times with PBS and collected for detection of intracellular ROS fluorescence by a fluorescence microscope (Olympus) with blue light motivating.

\section{Real-time PCR analysis}

Total RNA was extracted from cells by RNA kit II (Omega Bio-Tec Inc., USA) following manufacturer's instructions and quantified with Nano Drop spectrophotometry (BioTek, USA). We used $2 \mu \mathrm{g}$ total RNA to synthesize first strand cDNA by RevertAid first strand cDNA synthesis kit (Thermo Scientific). Real-time PCR was performed on BioRad CFX Connect ${ }^{\mathrm{TM}}$ Real-time System (Bio-Rad, USA) and amplified with an optimized cycling condition: $5 \mathrm{~min}$ at $95^{\circ} \mathrm{C}$, then $10 \mathrm{~s}$ at $95^{\circ} \mathrm{C}$ and $30 \mathrm{~s}$ at $60^{\circ} \mathrm{C}$ for 38 cycles. The total reaction system was $20 \mu \mathrm{L}: 10 \mu \mathrm{L} 2 \times$ QuantiFast SYBR Green PCR MasterMix (Qiagen, Germany), $1 \mu \mathrm{L}$ of cDNA templates, $0.5 \mu \mathrm{M}$ primer and $8 \mu \mathrm{L}$ of DNAase-RNase Free water. The primers were provided by Shenggong Company (Shanghai, China) and the sequences were used in our previous studies $(8,17)$.

\section{Cyp1a1 enzyme activity assay}

Twenty four hours after plasma treatment for different time, cells were harvested and washed with PBS for 3 times. Then the cyp1a1 enzyme activity was measured by P450Glo $^{\text {TM }}$ cyp1a1 Assay (Promega, USA) according to the manufacturer's instructions.

\section{Statistical analysis}

All values are presented as mean $\pm \mathrm{SD}$ of three independent experiments. Differences between groups were evaluated using one-way ANOVA and student $\mathrm{T}$ test. $\mathrm{P}<0.05$ was considered statistically significant.

\section{Results and discussion}

\section{Generation of He plasma by plasma jet}

In this study, we generated the plasma through a device modified by a needle. The profile of the plasma jet device is shown in Figure 1A. He plasma was powered by a stainless steel tube with a voltage of $10 \mathrm{kHz} / 8 \mathrm{kV}$, which was also used as $\mathrm{He}$ gas injection. He gas flow was regulated at a ratio of 2 SLM. Figure $1 B$ shows the discharging image of the He plasma jet.

\section{Detection of emission spectra}

Since there are many particles with high energy states in the plasma, by detecting their characteristic lines in the emission spectroscopy, the distribution of various particles in the plasma could be understood. The optical fiber was placed 1 and $2 \mathrm{~cm}$ away from the plasma jet and the emission spectra was shown in Figure 2. Several characteristic lines of N, $\mathrm{He}$ and $\mathrm{O}$ were marked in the spectra, and the emission intensity is negatively correlated with the distance from the plasma jet.

\section{Plasma enhance the sensitivity to bortezomib}

Bortezomib is a first line drug for MM clinical treatment, we investigated whether gas plasma has a synergistic effect with bortezomib in MM cells. The cell cytotoxicity of different concentration of bortezomib was tested by cell viability assay 24 and $48 \mathrm{~h}$ after co-incubation (Figure $3 A$ ). Meanwhile, the reduction of cell viability by plasma treatment for different durations was investigated 24 and $48 \mathrm{~h}$ after treatment (Figure $3 B$ ). We chose 1 and $3 \mathrm{nM}$ concentration of bortezomib and $30 \mathrm{~s}$ of plasma treatment for synergistic effects analysis. After $24 \mathrm{~h}$ co-treated with bortezomib and plasma, we found that $30 \mathrm{~s}$ of plasma treatment could both significantly enhance the sensitivity to 1 and $3 \mathrm{nM}$ of bortezomib treatment (Figure 3C).

\section{Plasma increased ROS levels}

Biological effects induced by plasma treatment were mostly related to ROS generation. We detected the extracellular and intracellular ROS accumulation after plasma treatment by micro-plate reader and fluorescence 


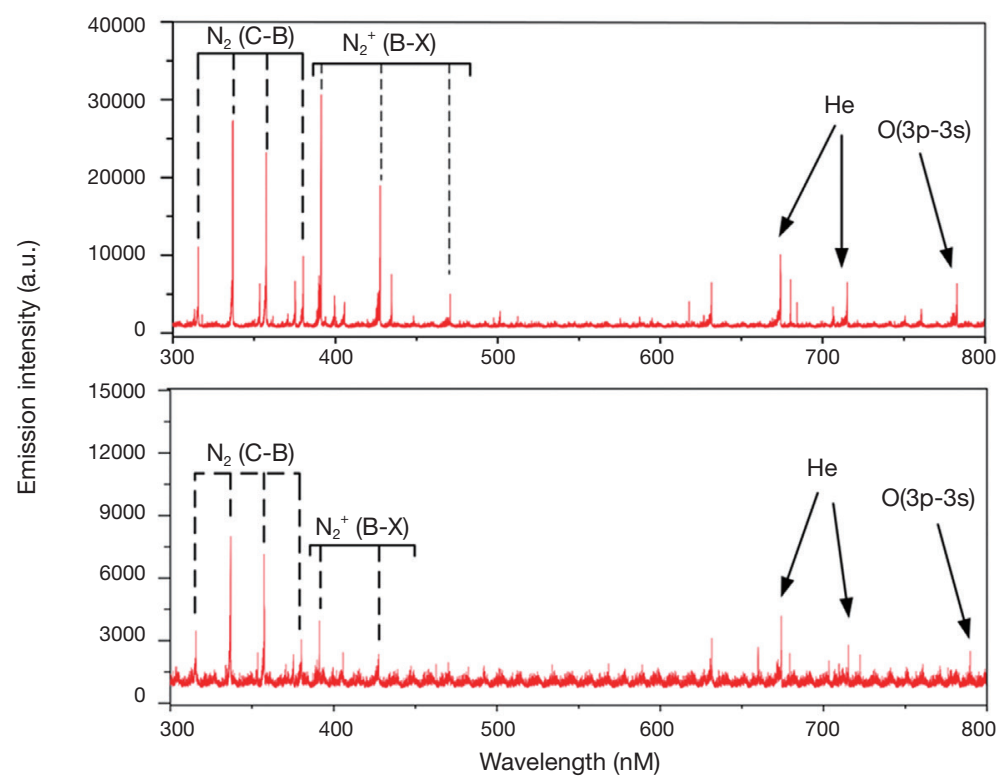

Figure 2 Typical optical emission spectra of the plasma ejected by APPJ at a distance of $1 \mathrm{~cm}$ (upper) and $2 \mathrm{~cm}$ (nether) from the nozzle.
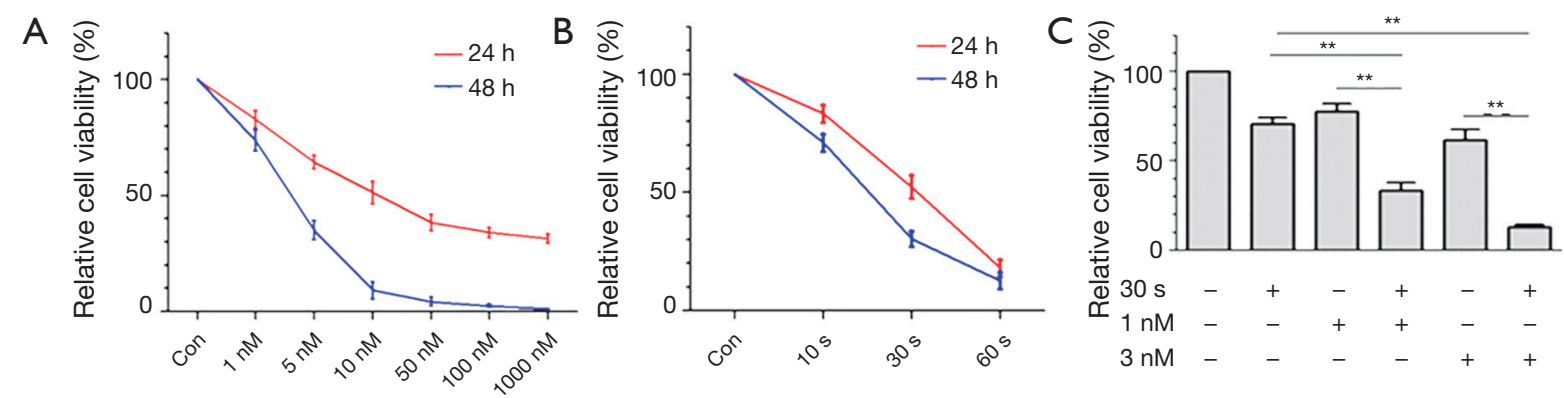

Figure 3 Plasma treatment enhance drug sensitivity to bortezomib in myeloma cells. (A) Cell viability was detected after treatment with different concentration of bortezomib for 24 and 48 h. (B) Analysis of cell viability 24 and 48 h after plasma treatment for 10,30 and 60 s. (C) Cell viability was determined $24 \mathrm{~h}$ after combination treatment of plasma and bortezomib. ${ }^{* *}$ indicates $\mathrm{P}<0.01$.

microscope. It showed that the extracellular ROS level was increased by plasma treatment in a time dependent manner, while the ROS scavenger (NAC) could prevent the ROS accumulation by plasma treatment (Figure $4 A$ ). The intracellular ROS was also increased after plasma treatment as the fluorescent intensity was higher than the control and NAC could reverse it (Figure 4B).

\section{Inbibition of Notch and cyp1a1 by plasma treatment}

In our previous study, we have demonstrated that cyp1a1, a
Cytochrome P450 enzyme for drug metabolism, is involved in bortezomib resistance in myeloma cells (8). Furthermore, Notch pathway is a critical signaling for regulating cyp1a1 activity. Therefore, we investigated whether plasma treatment had effects on these signaling. By real-time PCR we found that plasma treatment could significantly decrease the expression of Notch downstream target genes such as Hes1, Hes5, Hey1, Hey2 and HeyL (Figure 5A). Meanwhile, plasma treatment could down-regulate cyp1a1 expression in a time depend manner (Figure 5B). Besides, the enzyme activity assay showed that cyp1a1 activity was 
A

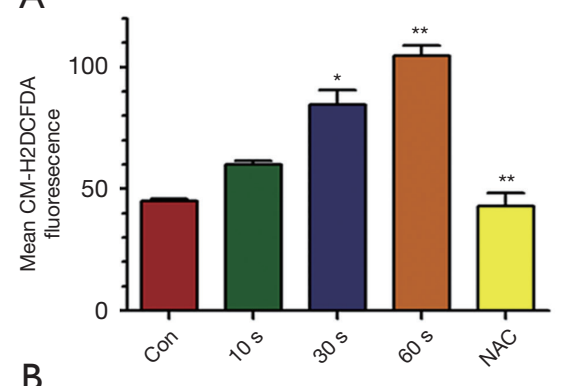

B

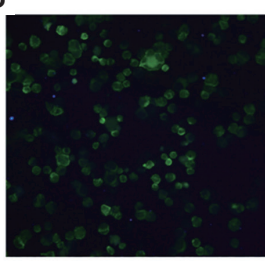

Con

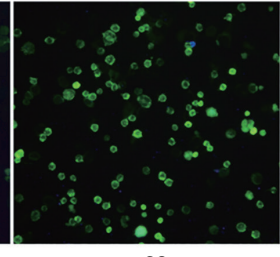

30 s

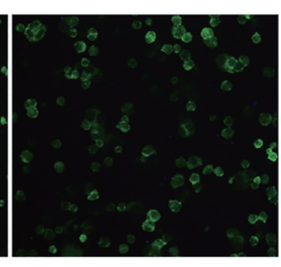

NAC
Figure 4 Plasma treatment increased ROS levels. (A) Extracellular ROS level was measured by microplate reader after plasma treatment. NAC is a general ROS scavenger. (B) Intracellular ROS was detected by fluorescent microscope after plasma treatment. *indicates $\mathrm{P}<0.05$, **indicates $\mathrm{P}<0.01$. suppressed by gas plasma treatment (Figure 5C).

\section{Conclusions}

Drug resistance is one of the major problems encountered in clinical therapy of multiple myeloma treatment. Bortezomib is a first line drug used in the standard treatment and has improved clinical outcome. However, some patients do not respond to bortezomib or they eventually relapse after response $(18,19)$. Besides, the 5 years overall survival is still not satisfied and the combination of several drugs to overcome the multiple drug resistance is applied. In this study, we first tried to couple two different kinds of treatment: the chemotherapy and the plasma treatment, to enhance the anti-tumor effects. Gas plasma is a newly developed technology that has been widely applied in biological and medical applications. We found that gas plasma treatment and bortezomib had a synergistic effect on the induction of myeloma cell death, which may lower the drug concentration to avoid the drug side effects in the future. In previous study we found that
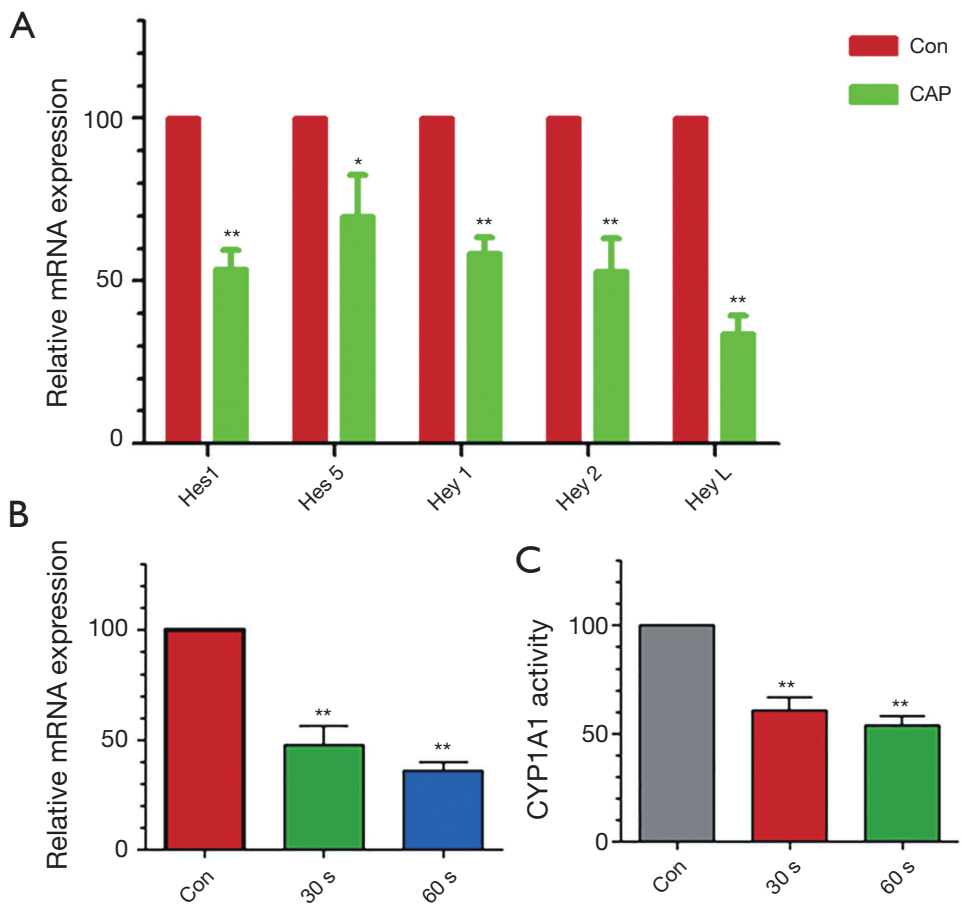

Figure 5 Inhibition of Notch and cyp1a1 by plasma treatment. (A) Expression of Notch target genes by real-time PCR 24 h after plasma treatment for $30 \mathrm{~s}$. (B) Expression of cyp1a1 by real-time PCR 24 h after plasma treatment for 30 s. (C) Measurement of cyp1a1 enzyme activity $24 \mathrm{~h}$ after plasma treatment for $30 \mathrm{~s}$. *indicates $\mathrm{P}<0.05$, **indicates $\mathrm{P}<0.01$. 
cyp1a1 up-regulated by Notch activation could induce drug resistance to bortezomib in myeloma cells (8), so we detected the Notch signaling and cyp1a1 expression after gas plasma treatment. Interesting, gas plasma treatment did suppress the Notch pathway and down-regulate the cyp1a1 expression and enzyme activity. But how the plasma interact with the Notch pathway remains unknown and it is an interesting work to be further investigated. As we know, plasma treatment induces various biological effects mostly through the generation of many ROS (11). It has been reported that Notch signaling could modulate ROS accumulation especially for $\mathrm{H}_{2} \mathrm{O}_{2}(20,21)$. To the contrary, ROS could also regulate Notch pathway and affected cell metabolism and cell death (22). In our results, ROS produced by gas plasma discharging could suppress the Notch downstream target genes. Kim et al. reported that ROS could down-regulate Notch signaling and induce cell death in breast cancer cells (23). Cao et al. demonstrated that inhibition of ROS production could up-regulate intracellular Notch and its downstream effectors (24). These results indicated that Notch might be a downstream target regulated by ROS. Furthermore, we found that cyp1a1, an enzyme involved in drug metabolism, was suppressed by plasma treatment induced Notch inhibition. By promoter analysis we found that there were several classical Notch CSL/RBP-J DNA binding sites (25) in the cypla1 promoter region (Supplementary) of either from human species or Mus musculus database, indicating that Notch could directly regulate cyp1a1 expression by modulating cpy1a1 mRNA transcription. Our results reported a new strategy to enhance the drug sensitivity by gas plasma treatment, which might be applied in clinical therapy to overcome drug resistance and to reduce side effects during chemotherapy.

In a whole, we used a new technology, the gas plasma, which could generate various ROS, to enhance the sensitivity of myeloma cells to bortezomib treatment. We further pointed out that plasma could inhibit cyp1a1 via Notch signaling and contribute to the synergistic effect with bortezomib.

\section{Acknowledgments}

Funding: This work is supported by the National Natural Science Foundation of China (Grant No. 51521065), First Class of China Postdoctoral Science Foundation (2017M610639) and Special Fund of China Postdoctoral Science Foundation, the Fundamental Research Funds for Central Universities, State Key Laboratory of Electrical
Insulation and Power Equipment (EIPE18312) and Special Fund of Shaanxi Postdoctoral Science Foundation (2017BSHTDZZ04).

\section{Footnote}

Conflicts of Interest: All authors have completed the ICMJE uniform disclosure form (available at http://dx.doi. org/10.21037/tcr.2019.10.43). The authors have no conflicts of interest to declare.

Ethical Statement: The authors are accountable for all aspects of the work in ensuring that questions related to the accuracy or integrity of any part of the work are appropriately investigated and resolved. Ethics approval was not required because no clinical experiments were involved in our paper.

Open Access Statement: This is an Open Access article distributed in accordance with the Creative Commons Attribution-NonCommercial-NoDerivs 4.0 International License (CC BY-NC-ND 4.0), which permits the noncommercial replication and distribution of the article with the strict proviso that no changes or edits are made and the original work is properly cited (including links to both the formal publication through the relevant DOI and the license). See: https://creativecommons.org/licenses/by-nc-nd/4.0/.

\section{References}

1. Kumar SK. Management of Multiple Myeloma. J Natl Compr Canc Netw 2018;16:624-7.

2. Krishnan SR, Jaiswal R, Brown RD, Luk F, Bebawy M. Multiple myeloma and persistence of drug resistance in the age of novel drugs. Int J Oncol 2016;49:33-50.

3. Podar K, Chauhan D, Anderson KC. Bone marrow microenvironment and the identification of new targets for myeloma therapy. Leukemia 2009;23:10-24.

4. Iida S. Mechanisms of action and resistance for multiple myeloma novel drug treatments. Int J Hematol 2016;104:271-2.

5. Nikesitch N, Ling SCW. Molecular mechanisms in multiple myeloma drug resistance. J Clin Pathol 2016;69:97-101.

6. Garavelli S, Lazzari E, Colombo M, et al. Multiple Myeloma-Associated Drug Resistance: Targeting the Notch Pathway. Haematologica 2015;100:159.

7. Milner LA. Notch signaling: a key to the pathogenesis of 
multiple myeloma? Blood 2004;103:3253-4.

8. Xu D, Hu J, De Bruyne E, et al. Dll1/Notch activation contributes to bortezomib resistance by upregulating CYP1A1 in multiple myeloma. Biochem Biophys Res Commun 2012;428:518-24.

9. Kong MG, Kroesen G, Morfill G, et al. Plasma medicine: an introductory review. New J Phys 2009;11:115012.

10. Fridman G, Friedman G, Gutsol A, et al. Applied plasma medicine. Plasma Process Polym 2008;5:503-33.

11. Graves DB. The emerging role of reactive oxygen and nitrogen species in redox biology and some implications for plasma applications to medicine and biology. J Phys D Appl Phys 2012;45:263001.

12. Xu D, Liu D, Wang B, et al. In Situ OH Generation from O2- and H2O2 Plays a Critical Role in Plasma-Induced Cell Death. PloS One 2015;10:e0128205.

13. Weltmann KD, Metelmann HR, von Woedtke T. Low temperature plasma applications in medicine. Europhysics News 2016;47:39-42.

14. Bekeschus S, Schmidt A, Weltmann KD, et al. The plasma jet kINPen-A powerful tool for wound healing. Clin Plasma Med 2016;4:19-28.

15. von Woedtke T, Reuter S, Masur K, et al. Plasmas for medicine. Phys Rep 2013;530:291-320.

16. Xu D, Wang B, Xu Y, et al. Intracellular ROS mediates gas plasma-facilitated cellular transfection in 2D and 3D cultures. Sci Rep 2016;6:27872.

17. Xu D, Hu J, Xu S, et al. Dll1/Notch activation accelerates multiple myeloma disease development by promoting

Cite this article as: Xu D, Cui Q, Xu Y, Chen Z, Xia W, Yang Y, Liu D. Plasma enhance drug sensitivity to bortezomib by inhibition of cyp1a1 in myeloma cells. Transl Cancer Res 2019;8(8):2841-2847. doi: 10.21037/tcr.2019.10.43
CD138+MM-cell proliferation. Leukemia 2012;26:1402-5.

18. Ludwig H, Beksac M, Blade J, et al. Current multiple myeloma treatment strategies with novel agents: a European perspective. Oncologist 2010;15:6-25.

19. Palumbo A, Rajkumar SV. Treatment of newly diagnosed myeloma. Leukemia. 2009;23:449-56.

20. Kamarehei M, Yazdanparast R. Modulation of notch signaling pathway to prevent $\mathrm{H} 2 \mathrm{O} 2 /$ menadione-induced SK-N-MC cells death by EUK134. Cell Mol Neurobiol 2014;34:1037-45.

21. Yu HC, Bai L, Yue SQ, et al. Notch signal protects nonparenchymal cells from ischemia/reperfusion injury in vitro by repressing ROS. Ann Hepatol 2013;12:815-21.

22. Caliceti C, Nigro P, Rizzo P, et al. ROS, Notch, and Wnt Signaling Pathways: Crosstalk between Three Major Regulators of Cardiovascular Biology. Biomed Res Int 2014;2014:318714.

23. Kim TH, Woo JS, Kim YK, et al. Silibinin Induces Cell Death through Reactive Oxygen Species-Dependent Downregulation of Notch-1/ERK/Akt Signaling in Human Breast Cancer Cells. J Pharmacol Exp Ther 2014;349:268-78.

24. Cao Y, Fang YX, Cai JY, et al. ROS functions as an upstream trigger for autophagy to drive hematopoietic stem cell differentiation. Hematology 2016;21:613-8.

25. Persson LM, Wilson AC. Wide-Scale Use of Notch Signaling Factor CSL/RBP-J kappa in RTA-Mediated Activation of Kaposi's Sarcoma-Associated Herpesvirus Lytic Genes. J Virol 2010;84:1334-47. 
>CYP1A1:chr15:72593795 [-2000-299] (-) [human, Homo sapiens] CATGCCCGGCTAATTTTTGTATTTTTGATAGAGATGGGGTTTTGCCATAT TGGCCAGGCTGGTCTTGAACTCCTGACCTCAGGCGATCCTCCCGACTCGG CCTTCCAAAGTCCTGGGATTACAGGCGTGAGCCACCGCACCTGCCCAGGT ACACTTTTTATCCAGATATCTATGCACATTGATTCTTGACTCCACACTCC TGCCTCTCTCCGCAAGGCCTTCCCTGACCCCCTTGTTTTGAATTGCAATC TGCCCTCACATAATCCGTACGCCTTTTCTGTTTAATTTTTTTCAACCGCA CTTTTTACCСTCTCACATTCTCTAGATTTGGGGCATATTACTTGTCTCCT TCCATCAGAATGTAAACTCCACGAGATTAGGAGTGCTGTCAGCTGTGTTC CCTTCTCTGTCAATCGCCAGCGCCTCCGAACAGCTCCTGGCACATAGTAG TTGCTTGGTAAATATTTGTGCAATGCACGAGGCCGCATATGACCGGAATC

GGTGAGGGGATTATTTTCTGGCCTGGACCAGCGACGGATGGTGGTGC CACCGGGTTGGGGAGCACGTCGGGGATGGCGCGTAACGATGTTAGCTGGG GCCAGGTTGAGCTAGGCACGCAAATACAACTTTTTTTTTCCTGGAAACCC TGTAACAGGAAGGTTCCGGAGGGCGGGACAGCGTCGGAGGCAGGCAGCTA GGCCATGCCAAATGGCACTGGGGCTTCGTGTCGTGCCACAGGCGTGGACC GAAAATGCGGACACATGCAGGCTGCCTCTCCTCGCAGGCAGAAGCCACAC GCAGACCTAGACCCTTTGCACCGCATCCCCTTATTCAATCGCGCACCCGC CACCCTTCGACAGTTCCTCTCCCTCCACCCCAACCCCACGCCGCGCGCGA GGCTGGCCCTTTAAGAGCCCCGCCCCGACTCCCTCCCCCCTCGCGTGACT GCGAGCCCCCGCGCCGGGCCGGGGAATGGGTCGGCTGGGTGGCTGCGCGG GCCTCCGGTCCTTCTCACGCAACGCCTGGGCACCGCGCCTCCGGGCCAGG TGGGGCGGGGACGGGCCGCCTGACCTCTGCCCCCTAGAGGGATGTCGCCG GCGCACGCAAGCTAGCCGGGGGTAGGGTGGGGGCTCCGCGCCAGGTGCCC CCTCCGTGGTCCCTGGGCCCGAGTCTTTCCGTGGCCCCCCGCCGCCGGAT TTCTGTGCTCTGCCAATCAAAGCACTAGCCACCCCGGGAGCCAAGAGGGA CCCTCAAGGGCCGGTGGGTCCTGGCTGGAGGGACCGCGCGTTGCAATCAG CACTAAGGCGATCCTAGAGGCTGCGAGGAGCCGCTAGTGAGCGCTCAGCG AGCCTGCCCCTTCGCCATCCATTCCGATCCTTCAATCAAGAGGCGCGAAC CTCAGCTAGTCGCCCGGGCTCTGGGGGACAGGTCCAGCCCCGCGGCGCCT CTGGCCTTCCGGCCCCCGTGACCTCAGGGCTGGGGTCGCAGCGCTTCTCA CGCGAGCCGGGACTCAGTAACCCCGGGAAGGAGGTCACCACGGGGCAGCC CCGCCCCCGCCTGCCGAGTCCTGGTAGGCTGTAGCGCTGGGGAGGCATCT GCACGCCCAGCGTTCCAGTGGGTGCAAAAATGACGAAGAGGAGTCCCCGC GCCCCAGGATGGAGCTTCCCGTACCCTCTCTTCGGGCTGTCCTGGGACTT CTCCCTCAAGCCCCCTCCTCGGCTGGGTTCTGCACTGCCCTTGGGACGCC TTGGAATTGGGACTTCCAGGTGTTCCCAGCCCTCACCCCTCTATGTACAG GCACCGAGATGTGTCCCATAGTGGGTTCTTGCCCACCCGACCCCCCACCC CCGCCGCCCTCCGCCACCTTTCTCTCCAATCCCAGAGAGACCAGCCCGGT TCAGGCTGCTTCTCCCTCCATCTCAGCTCGCTCCAGGGAAGGAGGCGTGG CCACACGTACAAGCCCGCCTATAAAGGTGGCAGTGCCTTCACCCTCACCC TGAAGGTGACAGTTCCTTGGAACCTTCCCTGATCCTTGTGATCCCAGGCT CCAAGAGTCCACCCTTCCCAGCTCAGCTCAGTACCTCAGGTGAGTTGCTG GGGGACTTCTGGCTTGCCCTTTCTCTCCCAATAAAAGGAACATTTTGGTG CCTCCAGGACTTCTTAGGTAGCTACCTGTCTAGCACCTCCAAAAAGGGAG GCTCAGAGTGTTTTTAGTGACCAGGCAGTCTAGCCCCCTAGTGGGGAAAC TGAGGCCAGGGGAAGAGGAGGACTTGCCCATGGTCCCACAGCTGGTACC 
1 [113-117] TGGGA

2 [498-502] TGGGA

3 [1692-1696] TGGGA

4 [1741-1745] TGGGA

5 [1757-1761] TGGGA

>Cyp1a1:chr9:57867391 [-2000-299](+) [mouse, Mus musculus]

AGACAACATCCTCACTACTGACTCTCATTTTCCTCGGTGGGATACTTCTT GAATAGGTAGACATGTCTGGAGAACACCACCCCCTTACCACAACCTCTAC ACATAACTAGAACATCTGGAAACACTGTGCCTATGAGGTGGGTCCTCTTA CCAGGAACATCCTCCAGGGAGATACCTGGGAGGCTTGACTGGACACTCTT GCCTCTGTTCAGTGCTATCTCAGAGAGCACTCCCCTGGCCACCTCATTTG CAGTTGTAAACTGTCCCCTGCATATCATTTATCTTTCTAGTGCTCTTTTT GTACTATGCCTGTCTCTGTGTTTTGTTACTTATCACCTGTTTCCCCCATA GCAGCGGGTAAGCTTCGTCAAAGGAGTATTGTTAATCCTTTTCACCTCTG CATCAATCACCAGCATCCAGCATAGTAGGAGCTTGGCGAATATTTGCACA ATGCATGAGGGCGGCATGTAACTAGAGTGGGAGGTGAGGGGGTGGATGAA GTGTTGTTTTTTTTTCCTGGTCTTGGTCACAGAGCAGATATCAATGTTCT TTCTGGGCTCGGGAGCTCACAGGGGAGGGCAGGTGAAGGTGTTAGTTAGG AACAGGTTGAGTTAGACACGCCAAGTTCAGATGTTCTTCTTACACTAATC TCACTCTGGGAGCACCCTTCAGGGCCAGAGAGCACCTGCAAAACAGCCAG CTAGGCGTGCCGGGTGGTGCTGCGTGTCCTGCCACTAAAAATGTGGACAC ACGCTGTTCAGGCCTTTGCTCTCAGGCAGAAGCCGAGCATCGCACGCAAA CCCGGCCCTTTGCACCGCTGGCGCTGTCTTGTCGCGCCCTTGCAAAGCAT AGACTATTTCTATCTCTTAAACCCCACCCCAACGCCCCAGGAGAGCTGGC CCTTTAAGAGCCTCACCCACGGTTCCCCTCCCCCAGCTAGCGTGACAGCA CTGGGACCCGCGCCCGGTTGTGAGTTGGGTAGCTGGGTGGCTGCGCGGGC CTCCAGGCTCTTCTCACGCAACTCCGGGGCACCTTGTCCCCAGCCAGGTG GGGCGGAGACAGGCAGCCCGACCTCTGCCCCCAGAGGATGGAGCAGGCTT ACGCACGCTAGCCTCAGGAACCTGTGTGCGTGCCAAGCATCACCCTTTGT AGCCCCAGACCCCCTCCTGCTGTCTCGCGTGGATCCTTCCTCCACCCTTT CCTCCACCATACTTAGATAGCTCTGCACCCGCCGCCCGACTTCTCTGTGT TGCCTGCTTCAGTATGTATGCACAACACTAGCAAGCCCCGGGAGCCTACA GGGAGCTGCAAGCGGGGACTCCCGGGCTTGCAGCTTGCCTAAGGTGACCC TTAGAGGTGAGATCTGCAAGCCTGCCATCCATCCCCACCCTCTAGATGAA GCAGCGCGAACTTCGGCCGATACCCAATTTGTGGGGCACAGAGTCAGTCC AATGGCGCCACTGGCCTTCCTGTCCTGTGACCTCTGGGCTGGGGTCGTTG CGCTTCTCACGCGAGCTTGGACTCAGTAATCCAGGGAAGCAAAGTCACCA CCCAGCTGTTCTCCCTCTACCAGCCTTTCCCGGGCACCCATTGGCTTGTA GTAGGCAAGAGGATCTTACACACTGAATGTTTCAGGGGTGCAAAAATAGT GAAAAGGAATCCCTATGACCCGGAATGGAGGCCCCAGTACTTACTTTTTA GGTTACCCCAGAATTTTTCCTCAAACCCCTCCCTCAGTGGGATTATGCAC TGTCCATGGAGCACCTTGAAAGTGTGGGGGGTGGTGACCCCAACCTTTAT TCTTTTTCTTTTCTTATGTTTTTTTGTGCCTGTACCTACATCAGGTATCC GGTATGGCTTCTTGCCTATCTCCCCCCTCCCCCCTGCCTAGAGCACTCCC TAAGGCTGTCCCTCCCTCGGTCCCACCACTGGGCTCAGATAAGGAGGCGT 
GGCCAACAGACACAGAGTCCTATAAAGGTGGTGGTGCCTTCACCCTAACC 2000 CTGAAGGTGGTAGTTCTTGGAGCTTCCCCGATCCTCCCTAGGGTCCTAGA 2050 GAACACTCTTCACTTCAGTCCCTCCTTACAGCCCAAGGTGAGTGCTTGGG 2100 GGGGCTCCTGGATTGCCССТСССТСССТСССТСССТСССТСССТСССТСС 2150 CAAGAAAAGGAAATTTTTGGTGAAGAGTTACTGGGTAGCTCCTTCAAAAC 2200 AGGGAACTACACTTTTATTTATTTATTTATTTATTTATTTAGTGACCAGG 2250 TAAATTCCTTAAGGGAGAAACTGAGGCCAGAGGTATCAGCTATTTAGAT 2299

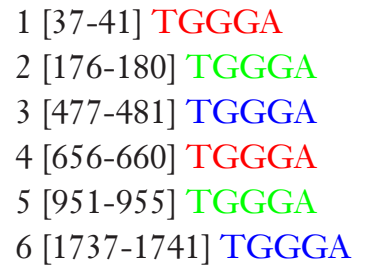

